\title{
Hierarchical Regulation of Centromeric Cohesion Protection by Meikin and Shugoshin during Meiosis I
}

\author{
Seira Miyazaki, ${ }^{1,2}$ Jihye Kim, ${ }^{3}$ Takeshi Sakuno, ${ }^{1,2}$ and Yoshinori Watanabe ${ }^{1,2}$ \\ ${ }^{1}$ Graduate Program in Biophysics and Biochemistry, Graduate School of Science, University of Tokyo, \\ 1-1-1Yayoi, Tokyo 113-0032, Japan \\ ${ }^{2}$ Laboratory of Chromosome Dynamics, Institute of Molecular and Cellular Biosciences, University \\ of Tokyo, 1-1-1Yayoi, Tokyo 113-0032, Japan \\ ${ }^{3}$ Research Institute, National Cancer Center, Goyang, Gyeonggi 410-769, Republic of Korea \\ Correspondence: ywatanab@iam.u-tokyo.ac.jp
}

\begin{abstract}
The kinetochore is the key apparatus regulating chromosome segregation. Particularly in meiosis, unlike in mitosis, sister kinetochores are captured by microtubules emanating from the same spindle pole (mono-orientation), and sister chromatid cohesion mediated by cohesin is protected at centromeres in the following anaphase. Shugoshin, which localizes to centromeres depending on the phosphorylation of histone $\mathrm{H} 2 \mathrm{~A}$ by Bub1 kinase, plays a central role in protecting meiotic cohesin Rec 8 from separase cleavage. Another key meiotic kinetochore factor, Moa1 (meikin), which was initially characterized as a monoorientation factor in fission yeast, also regulates cohesion protection. Moa1, which associates stably with CENP-C during meiosis I, recruits Plo1 (polo-like kinase) to the kinetochores and phosphorylates Spc7 (KNL1), inducing the persistent accumulation of Bub1 at kinetochores. The meiotic Bub1 pool ensures robust Sgol (shugoshin) localization and cohesion protection at centromeres by cooperating with heterochromatin protein Swi6, which binds and stabilizes Sgo1. Further, molecular genetic analyses reveal a hierarchical regulation of centromeric cohesion protection by meikin and shugoshin during meiosis I.
\end{abstract}

For the proper transmission of the genetic information, faithful chromosome segregation is essential in all organisms. During the cell cycle, sister chromatid cohesion is established in $\mathrm{S}$ phase dependent on the cohesin complex, and is maintained until metaphase. Sister chromatid cohesion at centromeres is essential to establish chromosome biorientation, in which sister kinetochores are captured by spindle microtubules from opposite poles. In the transition from metaphase to anaphase, the anaphase-promoting complex (APC) triggers the degradation of securin, an inhibitory chaperone for separase that cleaves cohesin and removes cohesin along the entire chromosome (Uhlmann et al. 1999; Onn et al. 2008; Peters et al. 2008; Nasmyth and Haering 2009). During meiosis, however, one round of DNA replication is followed by two consecutive nuclear divisions, resulting in the production of four haploid nuclei or gametes. In meiosis I, sister chromatids are captured from the same pole (mono-orientation), whereas homologous chromosomes (homologs) connected by chiasmata are captured by spindle microtubules emanating from the opposite poles (Fig. 1). At the onset of anaphase I, cohesin is cleaved by separase along the arm regions but protected at centromeres until metaphase II (Buonomo et al. 2000; Kitajima et al. 2003a; TachibanaKonwalski et al. 2010). Thus, mono-orientation and the protection of centromeric cohesion are two hallmarks of the regulation of meiotic chromosome segregation (Moore and Orr-Weaver 1998; Petronczki et al. 2003; Brar and Amon 2008; Watanabe 2012; Duro and Marston 2015).

\section{COHESION PROTECTION}

Cohesin complexes are modified in meiosis. Especially, the $\operatorname{Rad} 21$ (Scc1) subunit is largely replaced by its meiotic counterpart, Rec8 (Klein et al. 1999; Watanabe and Nurse 1999). During anaphase of meiosis I, Rec8 is cleaved only along the chromosome arms by separase, whereas centromeric Rec8 is preserved until meiosis II. If Rec8 is replaced by Rad21 during meiosis, sister chromatid cohesion, but not protection at the centromeres, is restored, leading to the separation of sister chromatids at meiosis I. Therefore, an intrinsic property of the Rec8 subunit absent from $\operatorname{Rad} 21$ contributes to centromeric protection at meiosis I (Toth et al. 2000; Yokobayashi et al. 2003). Pericentric heterochromatin plays a crucial role in enriching cohesin complexes and, thereby, strengthens centromeric cohesion in mitosis (Bernard et al. 2001b; Nonaka et al. 2002; Fukagawa et al. 2004). This might be applicable in meiosis, because the localization of the Rec8 complex is reduced from the pericentric regions in heterochromatin mutants (Kitajima et al. 2003b).

A functional screening of fission yeast identified the Rec8 protector as a gene that causes the disjunction of chromosomes, and thus it is toxic during mitotic growth only when co-expressed with $\operatorname{Rec} 8$ but not with $\operatorname{Rad} 21$ (Kitajima et al. 2004). This gene encodes a meiosis-specific protein named shugoshin (Sgo1), which means "guardian spirit" in Japanese. Sgo1 localizes exclusively at pericentric heterochromatin regions, the site at which

(C) 2017 Miyazaki et al. This article is distributed under the terms of the Creative Commons Attribution-NonCommercial License, which permits reuse and redistribution, except for commercial purposes, provided that the original author and source are credited. 
A

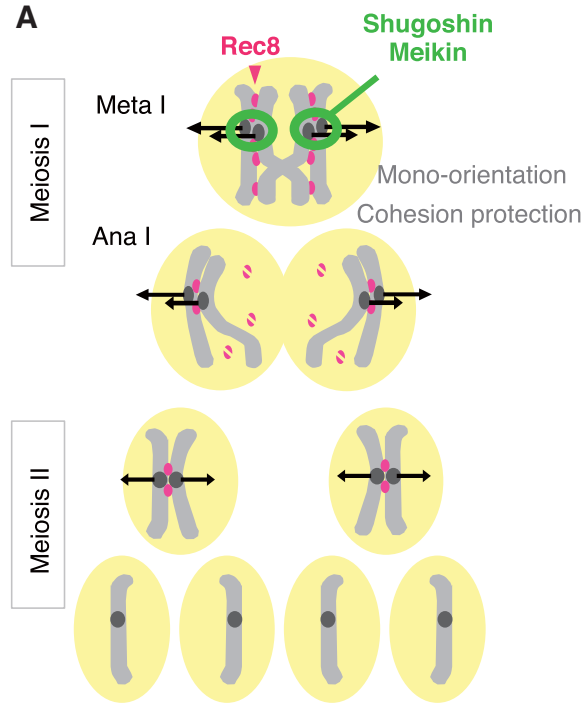

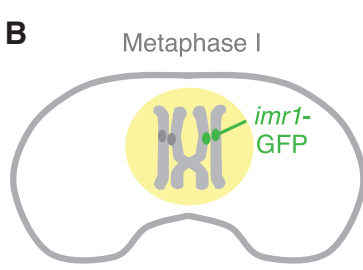

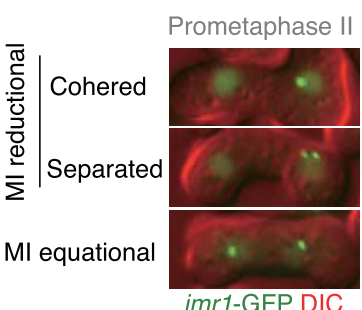

C imr1-GFP patterns at

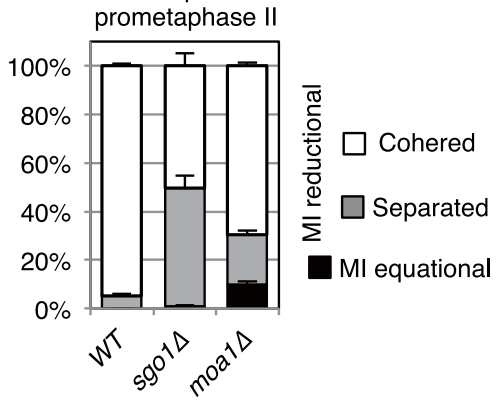

D

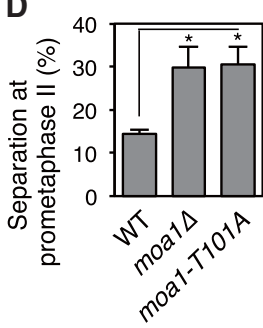

Figure 1. Moa1-Plo1 promotes cohesion protection at centromeres. (A) Schematic image of chromosome segregation during meiosis. $(B)$ The green dot represents an imrl-GFP-labeled centromere. In normal meiosis I (MI), sisters are captured by spindles from the same pole, and heterozygous imrl-GFP signals move to one side (reductional segregation). The majority of imrl-GFP signals in prometaphase II zygote are observed as one dot (cohered) because centromeric cohesion is protected, whereas some cells exhibit two dots (separated) because of the failure of cohesion protection. If mono-orientation is compromised at meiosis I, some sisters would segregate to opposite sides (equational segregation). $(C)$ The chromosome segregation pattern during meiosis I and the splitting of centromeres were counted by observing heterozygous imr l-GFP signals in prophase II arrest (by the mes 1-B44 mutation) in the indicated cells. Error bars, SD, $n>150$ cells, three independent experiments. $(D)$ The frequency of the splitting of centromeres was measured by observing heterozygous imrl-GFP signals in prometaphase II (prometa II) arrest (mesl) in the indicated cells. WT, wild type. (Modified, with permission, from Miyazaki et al. 2017, (c) John Wiley \& Sons, Inc.)

Rec8 was predicted to play a role in the centromeric protection at meiosis I (Kitajima et al. 2003b). Independent knockout screening in fission yeast and budding yeast also identified the sgo1/SGO1 gene (Marston et al. 2004; Rabitsch et al. 2004) as a Rec8 protector in meiosis. Remarkably, it turns out that shugoshin shares a hitherto unperceived limited similarity to MEI-S332, a Drosophila protein that was previously shown to be required for the persistence of centromeric cohesion during meiosis I (Davis 1971; Kerrebrock et al. 1995; Lee and Orr-Weaver 2001). Analyses in several eukaryotic organisms indicate that meiotic cohesin protection at centromeres is mediated by the centromeric protein shugoshin (Sgol in fission yeast). Shugoshin forms a complex with protein phosphatase 2A (PP2A) at the centromeres (Kitajima et al. 2006; Riedel et al. 2006; Tang et al. 2006; Lee et al. 2008; Llano et al. 2008) and antagonizes Rec8 phosphorylation, a prerequisite for cleavage by separase in meiosis I (Ishiguro et al. 2010; Katis et al. 2010).

Bub1, a well-conserved spindle checkpoint kinase, is required to preserve centromeric protection during meiosis I in fission yeast (Bernard et al. 2001a). Indeed, Sgo1 fails to localize at centromeres in bubl mutants (Kitajima et al. 2004). A biochemical approach in fission yeast identified serine 121 of histone $2 \mathrm{~A}(\mathrm{H} 2 \mathrm{~A})$ as a Bub1 substrate (Kawashima et al. 2010). Crucially, Sgo1 binds nucleosomes including phosphorylated H2A-S121, accounting for the mechanism of Sgol localization at centromeres (Kawashima et al. 2010). Sgol localization is also pro- moted by pericentric heterochromatin protein Swi6 (HP1), which binds directly to Sgol (Yamagishi et al. 2008). In mitosis, Bub1 as a complex with Bub3 localizes to kinetochores through its interaction with Spc7 (KNL1) only when Spc7 is phosphorylated by Mph1 (MPS1). Mph1 is a conserved protein kinase required for the spindle assembly checkpoint (SAC) locating at unattached or misaligned kinetochores (Yamagishi et al. 2012; London and Biggins 2014; Musacchio 2015; Sacristan and Kops 2015). Therefore, Bub1 is largely released from kinetochores when chromosomes are aligned during metaphase in mitotic cells. Recent reports reveal that KNL1 is also phosphorylated by PLK1 in humans and Caenorhabditis elegans (Espeut et al. 2015; von Schubert et al. 2015), suggesting the existence of a versatile regulation of Bub1 localization.

\section{MONO-ORIENTATION VERSUS BIORIENTATION}

The geometric aspect of kinetochores has been long recognized in vertebrates (Östergren 1951). The staining of human interphase nuclei with anticentromere antibodies revealed that the centromere is duplicated and resolved by the end of interphase (Brenner et al. 1981). This physical separation or resolution of sister centromeres would be important for the back-to-back assembly of sister kinetochores, thus facilitating bipolar attachment to microtubules in mitosis. In contrast to mitosis, cytological 
analyses of several animal germ cells have shown that sister kinetochores orient side by side and fuse in meiosis I (Goldstein 1981; Moore and Orr-Weaver 1998; Lee et al. 2000; Parra et al. 2004). The molecular mechanism underlying the regulation of kinetochore geometry has been studied recently (Watanabe 2012).

\section{Spo13-MONOPOLIN IN BUDDING YEAST}

In budding yeast a set of proteins called monopolin, which are required for mono-orientation, have been identified and extensively studied. Monopolin includes Csm1 (chromosome segregation in meiosis protein 1), Lrs4 (loss of rDNA silencing protein 4), Mam1 (monopolar microtubule attachment during meiosis I protein 1), and CK1, and localizes to centromeres specifically in meiosis I (Toth et al. 2000; Rabitsch et al. 2003). The structural analysis of Csm1-Lrs4 suggests that this complex forms a V shape with two pairs of kinetochore-binding domains that indeed bind kinetochore component Dns1. These results suggest that Csm1-Lrs4 may bring kinetochores together, which favors the clamp model (Corbett et al. 2010; Corbett and Harrison 2012). Evidence suggests that the enrichment of $\mathrm{CK} 1$ activity at kinetochores, which depends on the presence of Csm1-Lrs4, might be an ultimate requirement for the establishment of mono-orientation in budding yeast (Petronczki et al. 2006). However, which CK1 substrates are required for mono-orientation and the mechanism by which it occurs remain elusive. Budding yeast Spo13 (sporulation-specific protein 13), another factor that is required for mono-orientation as well as cohesion protection, associates with the Polo-like kinase Cdc5 and acts to recruit or stabilize the monopolin complex at centromeres (Clyne et al. 2003; Katis et al. 2004, 2010; Lee et al. 2004; Monje-Casas et al. 2007). In budding yeast, paired sister centromeres assemble a single kinetochore and bind only one microtubule (Sarangapani et al. 2014). Therefore, monopolin would conjoin two microtubule attachment sites and thereby make them into a one "point centromere."

\section{Rec8 IS REQUIRED FOR MONO- ORIENTATION IN FISSION YEAST}

In fission yeast, homologs of Csm1 and Lrs4 (named Pcs1 and Mde4, respectively) are dispensable for monoorientation in meiosis I. However, in mitosis, pscl or mde4 mutant cells show merotelic attachment, in which a single kinetochore is attached by microtubules emanating from both spindle poles (Gregan et al. 2007). Indeed, Pcs1-Mde4 recruits condensin, which may act to clamp together adjacent microtubule attachment sites, although the meiosis I-specific mono-orientation function is not conserved (Tada et al. 2011).

The finding that a rec 8 mutation in fission yeast causes equational, rather than reductional, division at meiosis I raises the possibility that cohesin complexes regulate kinetochore orientation. Similarly, plant and worm Rec8 have been shown to play an essential role in establishing monopolar attachment at meiosis I (Yu and Dawe 2000; Chelysheva et al. 2005; Severson et al. 2009). In fission yeast, a mitotic cohesin complex that includes $\operatorname{Rad} 21$ accumulates preferentially at the pericentromeric heterochromatin, whereas the meiotic Rec8-cohesin complex accumulates additionally at the core centromere, the region where the kinetochore assembles. When Rec8 is removed and replaced by $\operatorname{Rad} 21$ in meiosis I, the $\operatorname{Rad} 21-$ cohesin complex accumulates at the pericentromeric region, but much less at the central core region, causing equational rather than reductional division at meiosis I. When Rec8 is inactivated specifically only at the core centromere, but its other functions are preserved, kinetochores become bioriented at meiosis I, proving the essential role of Rec8 at the central core region for monoorientation (Yokobayashi and Watanabe 2005).

Based on the above evidence, it has been proposed that physical attachment of sister chromatids or cohesion at the centromeric core conjoins the two kinetochore domains at meiosis I, whereas the core regions open to opposite sides when not establishing this cohesion at mitosis and meiosis II. A direct observation of cohesion at the core centromere in fission yeast was enabled by popping out this DNA region from the neighboring chromosomal domains during prophase I, which is before the attachment of kinetochores to spindle microtubules (Sakuno et al. 2009). This analysis revealed that cohesion at the core centromere is indeed established and maintained, particularly during meiosis I, whereas this cohesion is lost in rec $8 \Delta$ or moal $\Delta$ (see below), which is defective in mono-orientation. Importantly, cohesion at the central core region is not detected during mitosis or meiosis II in wild-type cells, whereas cohesion at the pericentromeric region is intact. Finally, when a proteinous artificial tether is introduced at the core centromere, monopolar attachment is restored in meiotic rec $8 \Delta$ cells and even in normal mitotic cells. These results imply that mono-orientation of kinetochores is promoted ultimately by conjoining DNA duplexes underlying the kinetochores rather than the action of a kinetochore protein itself (Sakuno et al. 2009).

\section{CONSERVED MEIOTIC KINETOCHORE FACTOR MEIKIN (Moa1, Spo13, MEIKIN)}

Genetic screening to search for factors that regulate mono-orientation has identified a meiosis-specific kinetochore protein, Moa1 (monopolar attachment) in fission yeast (Yokobayashi and Watanabe 2005). Moal interacts with the conserved kinetochore protein Cnp1 (CENP-C homolog) and localizes exclusively at the central core of the centromere from prophase I to metaphase I but disappears in anaphase I (Tanaka et al. 2009). Moal also interacts with Rec8 and plays a role in establishing cohesion at the core centromere regions (mono-orientation) and some cohesion protection in pericentric regions. Taking advantage of the knowledge that Moal binds to conserved kinetochore protein CENP-C (Cnp3), two-hybrid screening using CENP-C as bait identified a meiosis-specific kinetochore protein MEIKIN in mice (Kim et al. 2015). Although there is no significant sequence homology 
between MEIKIN and Moa1, significant biochemical and functional similarities were identified between these two factors. Both MEIKIN and Moal recruit polo-like kinase (PLK) to kinetochores and the kinase activity of PLK is crucial for mono-orientation and cohesion protection (also see below). It turned out that these meiotic functions are reminiscent of those of budding yeast Spo13. Thus, the conserved meiosis-specific kinetochore regulator, meikin (MEIKIN in vertebrates; Moa1 in fission yeast; Spo13 in budding yeast) and its associated PLK play a crucial role in promoting mono-orientation and, at least partly, cohesion protection.

\section{FISSION YEAST MEIKIN AFFECTS COHESION PROTECTION}

Although fission yeast Moal was identified as a monoorientation factor, a role in cohesion protection was also implicated (Yokobayashi and Watanabe 2005). Indeed, in moal $\Delta$ cells, although a small population of cells undergo equational segregation at meiosis I (because of defects in mono-orientation), the majority undergo reductional segregation because of the presence of chiasmata and tension exerted across homologs (Fig. 1B). Strikingly, 22\% of these "reductional" moal $\Delta$ cells showed the separation of the GFP-marked centromere (imrl-GFP) in prometaphase II (Fig. 1B). This separation value is significantly higher than in wild-type cells $(<5 \%)$, although lower than in $s g o 1 \Delta$ cells $(50 \%)$, in which cohesion protection is completely abolished (Fig. 1B). These results suggest that moal $\Delta$ cells show partial defects in cohesion protection during reductional division at meiosis I.

Moal associates with Plol (PLK1 homolog), and the centromeric Plo1 is required for reductional segregation at meiosis I (Kim et al. 2015). Here we examined whether Plo1 is responsible for defects in cohesion protection at prometaphase II, as is observed in moals cells. For this purpose, we first analyzed the moal-T101A mutant, in which Moa1 localizes at kinetochores but fails to recruit Plo1 (Kim et al. 2015). Indeed, the separation of imr1GFP in prometaphase II was observed in moal-T101A cells to the same extent as in moal $\Delta$ cells (Fig. 1C). These results indicate that Plo1 recruited to kinetochores by Moa1 is responsible for the protection of centromeric cohesion during anaphase I.

\section{Bub1 REGULATES COHESION PROTECTION}

Bub1, a well-conserved spindle checkpoint kinase, is required to preserve centromeric protection during meiosis I (Bernard et al. 2001a). Indeed, Sgol fails to localize at centromeres in bub1 mutants (Kitajima et al. 2004; Fernius and Hardwick 2007). A biochemical approach in fission yeast identified serine 121 of histone 2A (H2A) as a Bub1 substrate (Kawashima et al. 2010). Crucially, Sgo1 binds nucleosomes, including phosphorylated H2A-S121, accounting for the mechanism of Sgol localization at centromeres (Kawashima et al. 2010). Sgol localization is also promoted by the pericentric heterochromatin protein Swi6
(HP1), which binds directly to Sgol (Yamagishi et al. 2008). In mitosis, Bub1 as a complex with Bub3 localizes to kinetochores through its interaction with Spc7 (KNL1) only when Spc7 is phosphorylated by Mph1 (MPS1) at unattached or misaligned kinetochores. Therefore, Bub1 is largely released from kinetochores when chromosomes are aligned during metaphase in mitotic cells.

\section{Moa1-Plo1 REGULATES MEIOTIC Bub1 LOCALIZATION}

KNL1 is also phosphorylated by PLK1 in humans and C. elegans (Espeut et al. 2015; von Schubert et al. 2015), suggesting the existence of a versatile regulation of Bub1 localization. It is reasonable to speculate that Moa1-Plo1, which is required for cohesion protection, may play a role in Bub1 enrichment during meiosis I. In fission yeast mitosis, Bub1 is enriched at kinetochores only when Mph1 (MPS1 homolog) accumulates at unattached kinetochores and phosphorylates the MELT repeats of the kinetochore protein Spc7 (KNL1) (Fig 2A; Yamagishi et al. 2012). Curiously, however, meiotic Bub1 signals are retained at kinetochores throughout metaphase I until anaphase I in wild-type cells, and the signals persist even in mph $1 \Delta$ cells (Fig. 2B). Instead, centromeric Bub1 signals decline around late metaphase I in moal $\Delta$ cells and largely disappear in mph $1 \Delta$ moal $\Delta$ cells (Fig. 2B). Thus, Moa1associated Plo1 may play a key role in the accumulation of Bub1 at kinetochores in a redundant capacity with Mph1. The time-lapse live cell imaging of Bub1-GFP indicates that the duration of metaphase I is shortened in moals

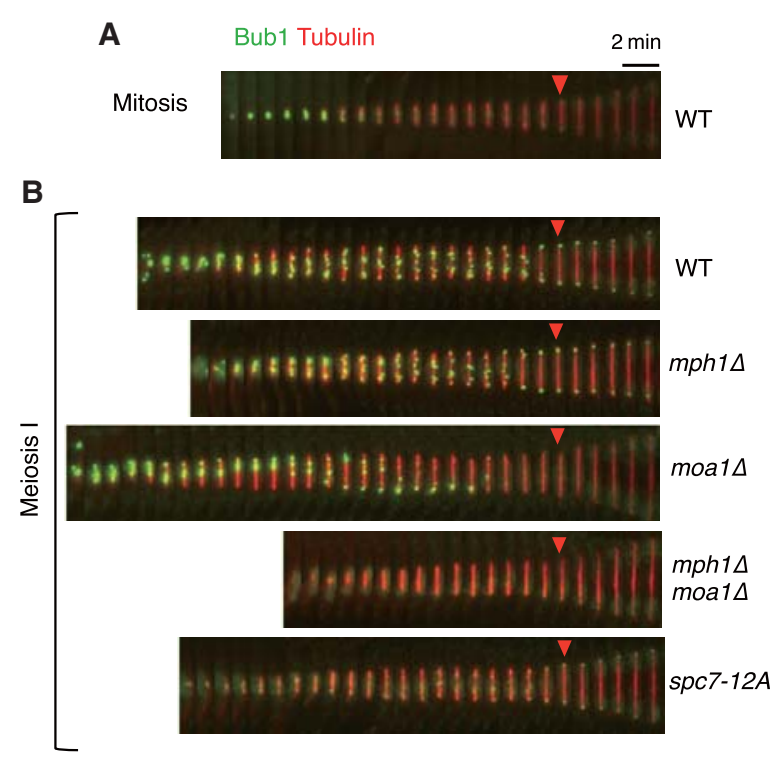

Figure 2. Bub1 is dispersed in mphl $\operatorname{moa} 1 \Delta$ cells as in $s p c 7$ $12 A$ cells. $(A, B)$ The indicated cells expressing Bub1-GFP and mCherry-Atb2 (tubulin) were observed by time-lapse imaging during mitosis $(A)$ or meiosis $\mathrm{I}(B)$ at 1-min intervals. A representative example of indicated cell is shown. The red arrowhead denotes the onset of spindle elongation at anaphase. WT, wild type. (Modified, with permission, from Miyazaki et al. 2017, (C) John Wiley \& Sons, Inc.) 
$m p h 1 \Delta$ cells (Fig. 2B), suggesting that Moa1-Plo1, like $\mathrm{Mph} 1$, has a function in SAC activation in meiotic cells.

\section{LIKE Mph1, Moa1-Plo1 PHOSPHORYLATES Spc7}

Is Moa1-Plo1 indeed responsible for the phosphorylation of MELT repeats in Spc7? Bacterially purified Plo1, like Mph1, phosphorylates the amino-terminal domain of Spc7 (Spc7-N), which contains MELT repeats, but not other domains that lack MELT repeats (Fig. 3A). Consistently, centromeric Bub1 signals are largely dispersed in $s p c 7-12 A$ cells as is seen in mphl 1 moal $\Delta$ cells during meiosis I (Fig. 2B). It is also shown that centromere-tethering Plo1 enables Bub1 recruitment to kinetochores even in mitotic interphase, and this is also the case in $m p h 1 \Delta$ cells (Miyazaki et al. 2017). These results strongly support the notion that meiosis-specific Bub1 recruitment to kinetochores relies on the phosphorylation of the MELT repeats on Spc7, a process mediated by Moa1-Plo1 and Mph1.

\section{KINETOCHORE-BOUND Bub1 AND CENTROMERIC HETEROCHROMATIN REDUNDANTLY SUSTAIN Sgo1 LOCALIZATION}

Notably, centromeric Sgol signals show an $\sim 70 \%$ reduction in $s p c 7-12 A$ cells, in which Bub1 is largely dispersed from kinetochores, whereas few defects in cohesion protection are observed in spc7-12A cells (Fig. 4A). Moreover, the expression of the Bub1 kinase catalyt-

A

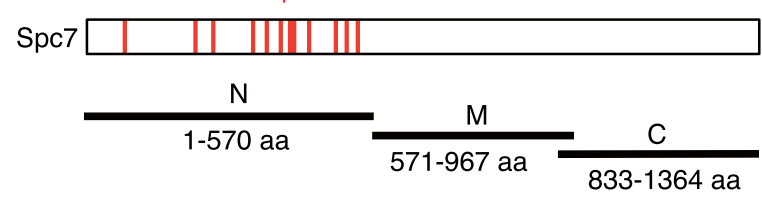

B

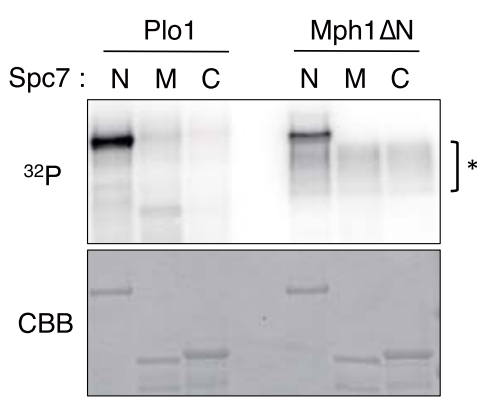

Figure 3. Spc7 phosphorylation at MELT sequence by Plo1 and Mph1 promotes Spc7 and Bub1/Bub3 binding. (A) Schematic depiction of Spc7 fragments used in the in vitro phosphorylation assay. (B) Recombinant GST-fused Spc7 fragments were incubated with recombinant GST-Plo1 (left) or GST-Mph1 $\Delta \mathrm{N}$ (kinase domain only) (right) in the presence of $\left[\gamma_{-}{ }^{32} \mathrm{P}\right]$ ATP. The incorporation of radioactive phosphate groups was visualized by autoradiography $\left({ }^{32} \mathrm{P}\right)$ and compared with protein levels (Coomassie brilliant blue $[\mathrm{CBB}])$. The asterisk indicates Mph $1 \Delta \mathrm{N}$ autophosphorylation. (Modified, with permission, from Miyazaki et al. 2017, (C) John Wiley \& Sons, Inc.) ic domain, which cannot bind kinetochores, allows Sgo1 localization at centromeres depending on the heterochromatin protein Swi6 (Kawashima et al. 2010). Therefore, the residual Sgol localization ( $\sim 30 \%$; yet functional) in $s p c 7-12 A$ cells might be produced by centromeric Swi6 together with dispersed Bub1 kinase activity, which would mildly phosphorylate histone $\mathrm{H} 2 \mathrm{~A}$ along the whole chromosomes including centromeres (Kawashima et al. 2010). Indeed, the cohesion protection defect in spc7$12 \mathrm{~A}$ cells is much enhanced by introducing the sgol-VE mutation, which renders Sgol unable to interact with Swi6 (Fig. 4A; Yamagishi et al. 2008). Thus, Bub1 enrichment mediated by Mph1 and Moa1-Plo1 (Spc7 phosphorylation) plays a crucial role in Sgo1 localization and cohesion protection in a redundant capacity with centromeric heterochromatin (Fig. 4B).

\section{Moa1-Plo1 MAY ENHANCE Sgo1 FUNCTION IN ADDITION TO ITS LOCALIZATION}

A remarkable observation is that protection defects are prominent in moal $\Delta$ cells but not in $s p c 7-12 A$ cells (Fig. 4C), whereas the opposite is true for Bub1 reduction (Fig. 2B). Therefore, in addition to the enhancement of Bub1 localization, Moa1 may play another key role in cohesion protection. This role of Moal might differ from the heterochromatin pathway because moal $\Delta$ and sgol-VE show additive defects in cohesion protection (Fig. 4C). Given that moals shows no additive defects with sgols (Fig, $4 \mathrm{C}$ ), it is reasonable to speculate that Moa1 may somehow enhance Sgol function directly as well as by its localization (Fig. 4B).

\section{CONCLUSION}

The mono-orientation of sister kinetochores and protection of centromeric cohesion are two hallmarks of the regulation of meiotic chromosome segregation that are widely conserved among eukaryotic organisms. Meikin is a recently emerging protein family that may regulate both mono-orientation and cohesion protection. Although fission yeast Moal was initially identified as a mono-orientation factor, it regulates cohesion protection similarly to mouse MEIKIN and budding yeast Spo13. Especially, in fission yeast, Moa1-Plo1 together with Mph1 play a crucial role in the enrichment of Bub1 at kinetochores throughout meiosis I. This Bubl pool ensures robust Sgo1 localization and cohesion protection at centromeres by cooperating with heterochromatin protein Swi6, which binds and stabilizes Sgo1. Although the meiosis-specific Bub1-Sgo1 enrichment mechanism is conserved in mouse (Miyazaki et al. 2017), the contribution of heterochromatin to Sgol stabilization is not yet proven in mammalian meiosis. In summary, accumulating evidence indicates that not only shugoshin but also meikin contribute to the meiosis I-specific cohesion protection mechanism. Previous studies suggest that the ectopic or physiological localization of shugoshin at centromeres in meiosis II cannot protect cohesin from separase cleavage at the onset of 


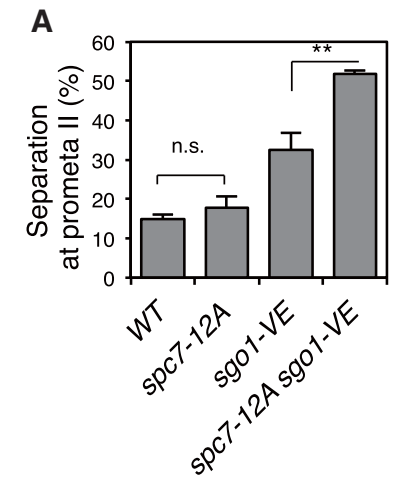

C

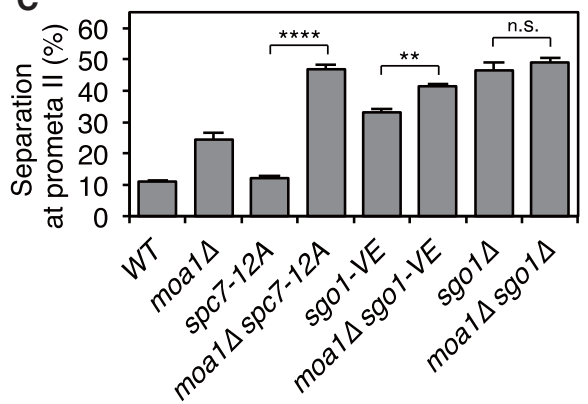

B

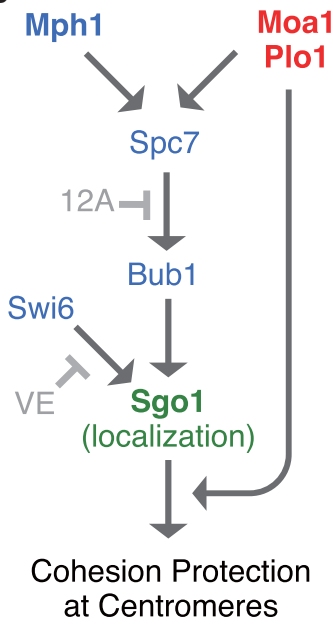

Figure 4. Moa1-Plo1 enhances Sgo1 function in addition to its localization. $(A, C)$ The frequency of centromere splitting was measured by observing heterozygous imrl-GFP signals in prometaphase II (prometa II) arrest (mes I) in the indicated cells. Error bars, SD from three independent experiments. Total cells: $n>140$. n.s.; not significant; **, $P<0.01$; *** $P<0.005$; ****, $P<0.001$; one-way ANOVA with Bonferroni's multiple comparisons test. $(B)$ Schematic depiction of Moal-Plol function in the regulation of cohesion protection at centromeres during meiosis I. Moa1-Plo1 facilitates Sgol localization at centromeres through Spc7 phosphorylation in a redundant capacity with Mph1. Swi6 stabilizes Sgol localization. Moa1-Plo1 also regulates another pathway required for cohesion protection in meiosis I. (Modified, with permission, from Miyazaki et al. 2017, (C) John Wiley \& Sons, Inc.)

anaphase II (Moore et al. 1998; Rabitsch et al. 2004). This could be accounted for at least in part, by the fact that meikin is absent from kinetochores in meiosis II.

\section{ACKNOWLEDGMENTS}

We thank all past members of the Watanabe laboratory for their work done in the laboratory and valuable discussion. This work was supported by MEXT KAKENHI grant number 25000014 (Y.W.).

\section{REFERENCES}

Bernard P, Maure JF, Javerzat JP. 2001a. Fission yeast Bub1 is essential in setting up the meiotic pattern of chromosome segregation. Nat Cell Biol 3: 522-526.

Bernard P, Maure JF, Partridge JF, Genier S, Javerzat JP, Allshire RC. 2001b. Requirement of heterochromatin for cohesion at centromeres. Science 294: 2539-2542.

Brar GA, Amon A. 2008. Emerging roles for centromeres in meiosis I chromosome segregation. Nat Rev Genet 9: 899910.

Brenner S, Pepper D, Berns MW, Tan E, Brinkley BR. 1981. Kinetochore structure, duplication, and distribution in mammalian cells: Analysis by human autoantibodies from scleroderma patients. J Cell Biol 91: 95-102.

Buonomo SB, Clyne RK, Fuchs J, Loidl J, Uhlmann F, Nasmyth K. 2000. Disjunction of homologous chromosomes in meiosis I depends on proteolytic cleavage of the meiotic cohesin Rec8 by separin. Cell 103: 387-398.
Chelysheva L, Daiallo S, Vezon D, Gendrot G, Vrielynck N, Belcram K, Rocques N, Marquez-Lema A, Bhatt AM, Horlow C, et al. 2005. AtREC8 and AtSCC3 are essential to the monopolar orientation of the kinetochores during meiosis. $J$ Cell Sci 118: 4621-4632.

Clyne RK, Katis VL, Jessop L, Benjamin KR, Herskowitz I, Lichten M, Nasmyth K. 2003. Polo-like kinase Cdc5 promotes chiasmata formation and cosegregation of sister centromeres at meiosis I. Nature Cell Biol 5: 480-485.

Corbett KD, Harrison SC. 2012. Molecular architecture of the yeast monopolin complex. Cell Rep 1: 583-589.

Corbett KD, Yip CK, Ee LS, Walz T, Amon A, Harrison SC. 2010. The monopolin complex crosslinks kinetochore components to regulate chromosome-microtubule attachments. Cell 142: 556-567.

Davis BK. 1971. Genetic analysis of a meiotic mutant resulting in precocious sister-centromere separation in Drosophila melanogaster. Mol Gen Genet 113: 251-272.

Duro E, Marston AL. 2015. From equator to pole: Splitting chromosomes in mitosis and meiosis. Gene Dev 29: 109-122.

Espeut J, Lara-Gonzalez P, Sassine M, Shiau AK, Desai A, Abrieu A. 2015. Natural loss of Mps1 kinase in nematodes uncovers a role for polo-like kinase 1 in spindle checkpoint initiation. Cell Rep 12: 58-65.

Fernius J, Hardwick KG. 2007. Bub1 kinase targets Sgol to ensure efficient chromosome biorientation in budding yeast mitosis. PLoS Genet 3: e213.

Fukagawa T, Nogami M, Yoshikawa M, Ikeno M, Okazaki T, Takami Y, Nakayama T, Oshimura M. 2004. Dicer is essential for formation of the heterochromatin structure in vertebrate cells. Nature Cell Biol 6: 784-791.

Goldstein LS. 1981. Kinetochore structure and its role in chromosome orientation during the first meiotic division in male D. melanogaster. Cell 25: 591-602. 
Gregan J, Riedel CG, Pidoux AL, Katou Y, Rumpf C, Schleiffer A, Kearsey SE, Shirahige K, Allshire RC, Nasmyth K. 2007. The kinetochore proteins Pcs1 and Mde4 and heterochromatin are required to prevent merotelic orientation. Curr Biol 17: $1190-1200$.

Ishiguro T, Tanaka K, Sakuno T, Watanabe Y. 2010. ShugoshinPP2A counteracts casein-kinase-1-dependent cleavage of Rec8 by separase. Nat Cell Biol 12: 500-506.

Katis VL, Matos J, Mori S, Shirahige K, Zachariae W, Nasmyth K. 2004. Spo13 facilitates monopolin recruitment to kinetochores and regulates maintenance of centromeric cohesion during yeast meiosis. Curr Biol 14: 2183-2196.

Katis VL, Lipp JJ, Imre R, Bogdanova A, Okaz E, Habermann B, Mechtler K, Nasmyth K, Zachariae W. 2010. Rec8 phosphorylation by casein kinase 1 and Cdc7-Dbf4 kinase regulates cohesin cleavage by separase during meiosis. Dev Cell 18: 397-409.

Kawashima SA, Yamagishi Y, Honda T, Ishiguro K, Watanabe Y. 2010. Phosphorylation of H2A by Bub1 prevents chromosomal instability through localizing shugoshin. Science 327: 172 177.

Kerrebrock AW, Moore DP, Wu JS, Orr-Weaver TL. 1995. MEIS332, a Drosophila protein required for sister-chromatid cohesion, can localize to meiotic centromere regions. Cell 83: 247-256.

Kim J, Ishiguro K, Nambu A, Akiyoshi B, Yokobayashi S, Kagami A, Ishiguro T, Pendas AM, Takeda N, Sakakibara Y, et al. 2015. Meikin is a conserved regulator of meiosis-I-specific kinetochore function. Nature 517: 466-471.

Kitajima TS, Miyazaki Y, Yamamoto M, Watanabe Y. 2003a. Rec8 cleavage by separase is required for meiotic nuclear divisions in fission yeast. EMBO J 22: 5643-5653.

Kitajima TS, Yokobayashi S, Yamamoto M, Watanabe Y. 2003b. Distinct cohesin complexes organize meiotic chromosome domains. Science 300: 1152-1155.

Kitajima TS, Kawashima SA, Watanabe Y. 2004. The conserved kinetochore protein shugoshin protects centromeric cohesion during meiosis. Nature 427: 510-517.

Kitajima TS, Sakuno T, Ishiguro K, Iemura S, Natsume T, Kawashima SA, Watanabe Y. 2006. Shugoshin collaborates with protein phosphatase $2 \mathrm{~A}$ to protect cohesin. Nature 441: 46-52.

Klein F, Mahr P, Galova M, Buonomo SBC, Michaelis C, Nairz K, Nasmyth K. 1999. A central role for cohesins in sister chromatid cohesion, formation of axial elements, and recombination during yeast meiosis. Cell 98: 91-103.

Lee BH, Kiburz BM, Amon A. 2004. Spo13 maintains centromeric cohesion and kinetochore coorientation during meiosis I. Curr Biol 14: 2168-2182.

Lee JY, Orr-Weaver TL. 2001. The molecular basis of sisterchromatid cohesion. Annu Rev Cell Dev Biol 17: 753-777.

Lee J, Miyano T, Dai Y, Wooding P, Yen TJ, Moor RM. 2000. Specific regulation of CENP-E and kinetochores during meiosis I/meiosis II transition in pig oocytes. Mol Reprod Dev 56: $51-62$.

Lee J, Kitajima TS, Tanno Y, Yoshida K, Morita T, Miyano T, Miyake M, Watanabe Y. 2008. Unified mode of centromeric protection by shugoshin in mammalian oocytes and somatic cells. Nature Cell Biol 10: 42-52.

Llano E, Gomez R, Gutierrez-Caballero C, Herran Y, SanchezMartin M, Vazquez-Quinones L, Hernandez T, de Alava E, Cuadrado A, Barbero JL, et al. 2008. Shugoshin-2 is essential for the completion of meiosis but not for mitotic cell division in mice. Genes Dev 22: 2400-2413.

London N, Biggins S. 2014. Signalling dynamics in the spindle checkpoint response. Nat Rev Mol Cell Biol 15: 736-748.

Marston AL, Tham WH, Shah H, Amon A. 2004. A genomewide screen identifies genes required for centromeric cohesion. Science 303: 1367-1370.

Miyazaki S, Kim J, Yamagishi Y, Ishiguro T, Okada Y, Tanno Y, Sakuno T, Watanabe Y. 2017. Meikin-associated polo-like kinase specifies Bub1 distribution in meiosis I. Genes Cells 22: $552-567$.
Monje-Casas F, Prabhu VR, Lee BH, Boselli M, Amon A. 2007. Kinetochore orientation during meiosis is controlled by Aurora $\mathrm{B}$ and the monopolin complex. Cell 128: 477-490.

Moore DP, Orr-Weaver TL. 1998. Chromosome segregation during meiosis: Building an unambivalent bivalent. Curr Top Dev Biol 37: 263-299.

Moore DP, Page AW, Tang TT, Kerrebrock AW, Orr-Weaver TL. 1998. The cohesion protein MEI-S332 localizes to condensed meiotic and mitotic centromeres until sister chromatids separate. J Cell Biol 140: 1003-1012.

Musacchio A. 2015. The molecular biology of spindle assembly checkpoint signaling dynamics. Curr Biol 25: R1002-R1018.

Nasmyth K, Haering CH. 2009. Cohesin: Its roles and mechanisms. Annu Rev Genet 43: 525-558.

Nonaka N, Kitajima T, Yokobayashi S, Xiao G, Yamamoto M, Grewal SI, Watanabe Y. 2002. Recruitment of cohesin to heterochromatic regions by Swi6/HP1 in fission yeast. Nat Cell Biol 4: 89-93.

Onn I, Heidinger-Pauli JM, Guacci V, Unal E, Koshland DE. 2008. Sister chromatid cohesion: A simple concept with a complex reality. Annu Rev Cell Dev Bi 24: 105-129.

Östergren G. 1951. The mechanism of co-orientation in bivalents and multivalents. Hereditas 37: 85-156.

Parra MT, Viera A, Gomez R, Page J, Benavente R, Santos JL, Rufas JS, Suja JA. 2004. Involvement of the cohesin Rad21 and SCP3 in monopolar attachment of sister kinetochores during mouse meiosis I. J Cell Sci 117: 1221-1234.

Peters JM, Tedeschi A, Schmitz J. 2008. The cohesin complex and its roles in chromosome biology. Genes Dev 22: 3089 3114.

Petronczki M, Siomos MF, Nasmyth K. 2003. Un menage a quatre: The molecular biology of chromosome segregation in meiosis. Cell 112: 423-440.

Petronczki M, Matos J, Mori S, Gregan J, Bogdanova A, Schwickart M, Mechtler K, Shirahige K, Zachariae W, Nasmyth K. 2006. Monopolar attachment of sister kinetochores at meiosis I requires casein kinase 1. Cell 126: 1049-1064.

Rabitsch KP, Petronczki M, Javerzat JP, Genier S, Chwalla B, Schleiffer A, Tanaka TU, Nasmyth K. 2003. Kinetochore recruitment of two nucleolar proteins is required for homolog segregation in meiosis I. Dev Cell 4: 535-548.

Rabitsch KP, Gregan J, Schleiffer A, Javerzat JP, Eisenhaber F, Nasmyth K. 2004. Two fission yeast homologs of Drosophila Mei-S332 are required for chromosome segregation during meiosis I and II. Curr Biol 14: 287-301.

Riedel CG, Katis VL, Katou Y, Mori S, Itoh T, Helmhart W, Galova M, Petronczki M, Gregan J, Cetin B, et al. 2006. Protein phosphatase $2 \mathrm{~A}$ protects centromeric sister chromatid cohesion during meiosis I. Nature 441: 53-61.

Sacristan C, Kops GJ. 2015. Joined at the hip: Kinetochores, microtubules, and spindle assembly checkpoint signaling. Trends Cell Biol 25: 21-28.

Sakuno T, Tada K, Watanabe Y. 2009. Kinetochore geometry defined by cohesion within the centromere. Nature 458: 852-858.

Sarangapani KK, Duro E, Deng Y, Alves FD, Ye Q, Opoku KN, Ceto S, Rappsilber J, Corbett KD, Biggins S, et al. 2014. Sister kinetochores are mechanically fused during meiosis I in yeast. Science 346: 248-251.

Severson AF, Ling L, van Zuylen V, Meyer BJ. 2009. The axial element protein HTP-3 promotes cohesin loading and meiotic axis assembly in $C$. elegans to implement the meiotic program of chromosome segregation. Genes Dev 23: 1763-1778.

Tachibana-Konwalski K, Godwin J, van der Weyden L, Champion L, Kudo NR, Adams DJ, Nasmyth K. 2010. Rec8-containing cohesin maintains bivalents without turnover during the growing phase of mouse oocytes. Genes Dev 24: 2505 2516.

Tada K, Susumu H, Sakuno T, Watanabe Y. 2011. Condensin association with histone H2A shapes mitotic chromosomes. Nature 474: 477-483.

Tanaka K, Chang HL, Kagami A, Watanabe Y. 2009. CENP-C functions as a scaffold for effectors with essential kineto- 
chore functions in mitosis and meiosis. Dev Cell 17: 334 343.

Tang Z, Shu H, Qi W, Mahmood NA, Mumby MC, Yu H. 2006. PP2A is required for centromeric localization of Sgol and proper chromosome segregation. Dev Cell 10: 575-585.

Toth A, Rabitsch KP, Galova M, Schleiffer A, Buonomo SB, Nasmyth K. 2000. Functional genomics identifies monopolin: A kinetochore protein required for segregation of homologs during meiosis I. Cell 103: 1155-1168.

Uhlmann F, Lottspeich F, Nasmyth K. 1999. Sister-chromatid separation at anaphase onset is promoted by cleavage of the cohesin subunit Scc1. Nature 400: 37-42.

von Schubert C, Cubizolles F, Bracher JM, Sliedrecht T, Kops GJPL, Nigg EA. 2015.Plk1 and Mps1 cooperatively regulate the spindle assembly checkpoint in human cells. Cell Rep 12: 66-78.

Watanabe Y. 2012. Geometry and force behind kinetochore orientation: Lessons from meiosis. Nat Rev Mol Cell Biol 13: 370-382.
Watanabe Y, Nurse P. 1999. Cohesin Rec8 is required for reductional chromosome segregation at meiosis. Nature 400: 461464.

Yamagishi Y, Sakuno T, Shimura M, Watanabe Y. 2008. Heterochromatin links to centromeric protection by recruiting shugoshin. Nature 455: 251-255.

Yamagishi Y, Yang CH, Tanno Y, Watanabe Y. 2012. MPS1/ Mph1 phosphorylates the kinetochore protein KNL1/Spc7 to recruit SAC components. Nat Cell Biol 14: 746-752.

Yokobayashi S, Watanabe Y. 2005. The kinetochore protein Moa1 enables cohesion-mediated monopolar attachment at meiosis I. Cell 123: 803-817.

Yokobayashi S, Yamamoto M, Watanabe Y. 2003. Cohesins determine the attachment manner of kinetochores to spindle microtubules at meiosis I in fission yeast. Mol Cell Biol 23: 3965-3973.

Yu H-G, Dawe RK. 2000. Functional redundancy in the maize meiotic kinetochore. J Cell Biol 151: 131-141. 


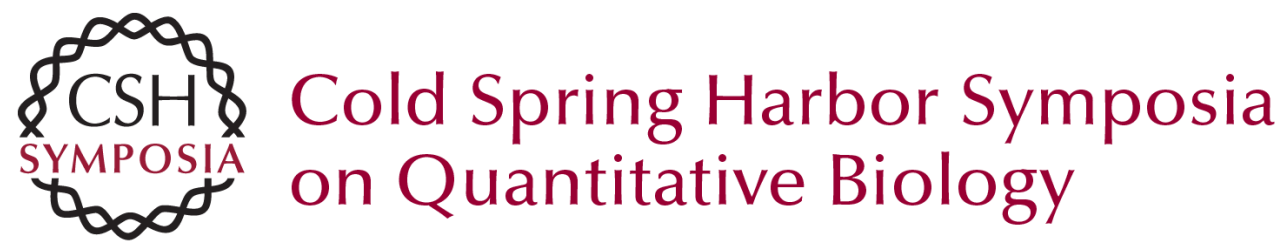

\section{Hierarchical Regulation of Centromeric Cohesion Protection by Meikin and Shugoshin during Meiosis I}

Seira Miyazaki, Jihye Kim, Takeshi Sakuno, et al.

Cold Spring Harb Symp Quant Biol 2017 82: 259-266 originally published online December 1, 2017

Access the most recent version at doi:10.1101/sqb.2017.82.033811

References This article cites 68 articles, 17 of which can be accessed free at: http://symposium.cshlp.org/content/82/259.full.html\#ref-list-1

Creative This article is distributed under the terms of the

Commons http://creativecommons.org/licenses/by-nc/4.0/, which permits reuse and

License redistribution, except for commercial purposes, provided that the original author and source are credited.

Email Alerting Receive free email alerts when new articles cite this article - sign up in Service the box at the top right corner of the article or click here. 